\title{
Laryngeal Amyloidosis: A Decadal Experience
}

\author{
Arya Raviraj ${ }^{1}$, Jayakumar R Menon ${ }^{2}$, Amrita S Mathew ${ }^{3}$
}

\begin{abstract}
Aim and objective: To determine the demographic and clinical characteristics, disease type, and appropriate treatment for laryngeal amyloidosis. Materials and methods: A retrospective descriptive study was conducted which included the data of 16 patients who had a laryngeal lesion which was excised and diagnosed as amyloidosis on histopathological examination, evaluated for systemic disease, and followed up. Demographic details, symptomatology, videolaryngoscopic examination findings, disease type, and treatment modality were analyzed.

Results: The male:female ratio was 1:1.29. The mean age of patients was $35.19 \pm 6.76$ years, with $87.5 \%$ aged between 30 years and 40 years. The mean duration of symptoms was $5.44 \pm 4.53$ months, ranging from 2 to 12 months. Symptoms included hoarseness of voice (62.5\%) and dyspnea (37.5\%). Lesions were located in the supraglottis (50\%), subglottis (37.5\%), and glottis (12.5\%). Macroscopically, $25 \%$ of lesions were polypoid and $75 \%$ were infiltrative. Microlaryngeal excision of the lesion was performed using cold steel instruments in $68.75 \%$ of cases and coblation assisted in $31.25 \%$ of cases. On immunohistochemistry, $93.75 \%$ were AL type and $6.25 \%$ were AA type. All cases were the primary localized type. All patients were followed up regularly. One recurrence and thus revision surgery aided with laser was recorded.

Conclusion: The diagnosis of laryngeal amyloidosis requires a high index of suspicion based on its clinical features. The localized laryngeal disease can be successfully treated by endoscopic excision of the lesion using either cold steel instrumentation, laser or coblation, as was performed in our study with favorable results. The importance of continued long-term follow-up is emphasized in view of a high predilection for recurrence in amyloidosis.
\end{abstract}

Keywords: Airway obstruction/pathology, Amyloidosis/surgery, Hoarseness/etiology, Laryngeal diseases/surgery, Laryngoscopy, Larynx/ pathology.

International Journal of Phonosurgery \& Laryngology (2021): 10.5005/jp-journals-10023-1197

\section{INTRODUCTION}

Amyloidosis is an uncommon disease of unknown etiology and slow progression. It is characterized by extracellular proteinaceous deposition of an abnormal insoluble group of fibrils which gives it its hallmark microscopic, histochemical, and ultrastructural attributes. ${ }^{1,2}$ There are multiple ways of classifying amyloidosis such as the etiological classification into hereditary, idiopathic/primary and secondary/reactive, clinical classification into localized and systemic types, and the immunohistochemical classification into numerous types based on the type of protein deposited, each with its prognostic ramifications. ${ }^{3,4}$

Any organ in the body can be affected by amyloidosis. The most common sites of involvement in systemic amyloidosis are the kidneys and heart, while in localized amyloidosis, the upper and lower airway, eye, genitourinary tract, and skin are commonly affected. Clinical manifestations of amyloidosis are related to the organ/site involved. ${ }^{5}$

In the head and neck region, the larynx is the most commonly involved site in cases with isolated amyloid deposits. ${ }^{2}$ Laryngeal amyloidosis has been described as a chronic, insidious, progressive, recurrent disease characterized by hoarseness, dyspnea, and stridor, accompanied by characteristic polypoidal or subepithelial infiltrating lesions, with a pinkish smooth surface and a distinctive yellowish hue on laryngeal visualization. ${ }^{6}$ A diagnostic dilemma can arise from its ability to mimic the appearance of a myriad of other laryngeal lesions clinically, which can be definitively distinguished by specialized histopathological examination. ${ }^{7}$ Management of localized lesions encompasses various methods of endoscopic excision with follow-up. ${ }^{8}$ This study was undertaken to determine the demographic and clinical characteristics, disease type, and
${ }^{1-3}$ Department of Laryngology, Dr Jayakumar's Institute of Laryngology, Thiruvananthapuram, Kerala, India

Corresponding Author: Arya Raviraj, Department of Laryngology, Dr Jayakumar's Institute of Laryngology, Thiruvananthapuram, Kerala, India, Phone: +91 8547073871, e-mail: aryaraviraj@gmail.com

How to cite this article: Raviraj A, Menon JR, Mathew AS. Laryngeal Amyloidosis: A Decadal Experience. Int J Phonosurg Laryngol 2021;11(1):5-9.

Source of support: Nil

Conflict of interest: None

pertinent treatment for this rare clinical disorder of laryngeal amyloidosis.

\section{Materials and Methods}

Data from the electronic medical records of patients who presented to the Department of Laryngology at our tertiary center between the years 2010 and 2019 were reviewed for the following criteria in a retrospective descriptive study. Patients included were those who were identified on videolaryngoscopic examination with a laryngeal lesion, for which they underwent definitive surgical intervention, were thereafter diagnosed with amyloidosis on histopathological examination of the excised tissue, were appropriately evaluated for systemic disease, and thereafter followed up at regular intervals. Patients with other laryngeal pathology, who declined investigations or treatment, were lost to follow-up, or had incomplete data/records were excluded.

Demographic details, symptomatology, videolaryngoscopic examination findings, disease type, and treatment modality were 
tabulated and apposite descriptive and inferential statistical analysis was performed. Results on continuous measurements were presented as mean \pm standard deviation and results on categorical measurements were presented as number (frequency) and percentage. Parametric data were analyzed using the one-way ANOVA test and non-parametric data were analyzed using Fisher's exact test. $p<0.05$ was considered as significant.

\section{Results}

All of the 16 patients met the inclusion criteria. The male:female ratio was $1: 1.29$ with $43.75 \%$ of males and $56.25 \%$ of females. The mean age of patients was $35.19 \pm 6.76$ years ranging from 20 to 55 years, with $87.5 \%$ aged between 30 years and 40 years and $6.25 \%$ each aged between $20-30$ and $50-60$ years. The mean duration of symptoms was $5.44 \pm 4.53$ months. The presenting symptoms were hoarseness of voice in $62.5 \%$ and dyspnea in $37.5 \%$ of cases.

Examination revealed lesions in various subsites of the larynx (Fig. 1). All supraglottic lesions involved the false cord (100\%). Within the glottis, lesions were observed involving the true vocal cord in $50 \%$ and the anterior commissure in $50 \%$. Overall, $43.75 \%$ of lesions were unilateral while the remaining $56.25 \%$ were bilateral. Macroscopically, 25\% of lesions were polypoid and $75 \%$ were infiltrative. On immunohistochemical analysis, 93.75\% were AL type and $6.25 \%$ were AA type.

Results of analysis of disease characteristics based on the anatomical location of the lesion within the larynx were tabulated (Table 1). There was no significant difference in the mean age of individuals in each subsite affected ( $p=0.943$ ). Supraglottic lesions were identified more commonly in males, glottic lesions equally in both sexes, and subglottic lesions in females, the result being significant ( $p=0.092$ ). Hoarseness of voice was significantly greater among those with supraglottic and glottic lesions, while dyspnea was greater among those with subglottic lesions ( $p=$ 0.00012 ; significant). The duration of these symptoms was found to be significantly shorter among those with subglottic lesions than in supraglottic and glottic lesions ( $p=0.00942$ ). Unilateral lesions were significantly more common in the supraglottis and glottis while bilateral lesions were more frequent in the subglottis $(p=0.0118)$. Macroscopically, infiltrative types of lesions were significantly more frequent in the subglottis than in other sites, whereas polypoid lesions were most commonly identified in the glottis $(p=0.03187)$.

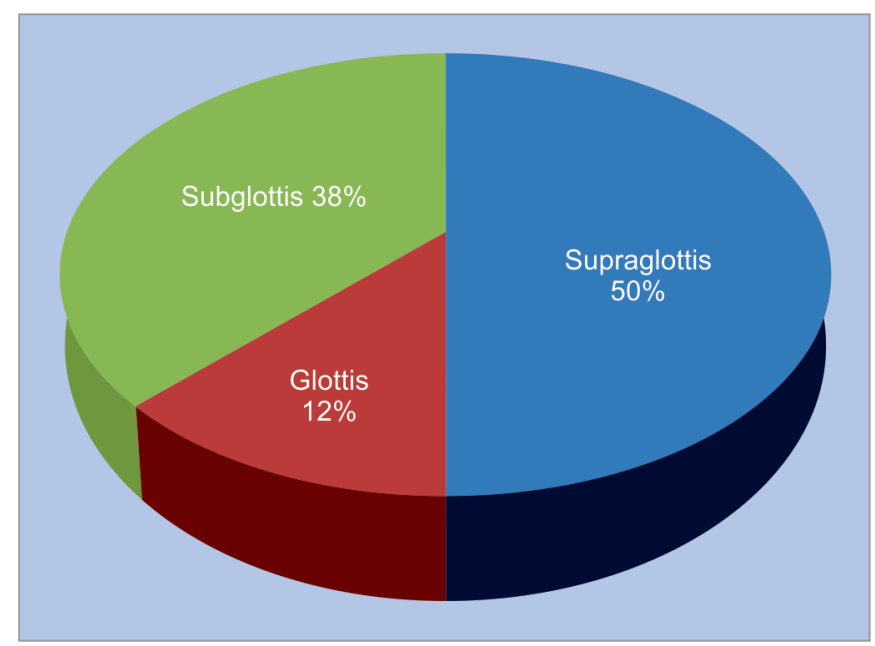

Fig. 1: Anatomical distribution of lesions in laryngeal amyloidosis
An equivalent result was observed with respect to the protein type identified on immunohistochemistry.

Microlaryngeal excision of the lesion was performed using cold steel instruments in $68.75 \%$ of cases and coblation assisted in $31.25 \%$ of cases (Fig. 2). All cases with subglottic involvement had bilateral lesions and hence surgical excision was performed in two stages (at 3 months intervals; both stages together were considered as one intervention). No complications were encountered. All patients had subjective improvement of voice. Systemic involvement was ruled out following relevant clinical, biochemical, pathological, and radiological evaluation in $100 \%$ of cases. All patients underwent regular follow-up. On follow-up, one case with subglottic lesions presented with a recurrence of signs and symptoms 6 months following complete excision (cold steel microlaryngoscopy was used, as other modalities like laser or coblator was not available in the department at that time) and thereafter underwent revision diode laser-assisted microlaryngoscopic excision following which no further recurrence was observed.

\section{Discussion}

Amyloidosis is a polymorphic conglomerate of disorders caused by the extracellular deposition of aberrant fibrillar proteins in various organs of the body. ${ }^{9}$ Von Rokitansky was the first to discover this protein in the spleen and liver of a human cadaver in 1842. In 1851, Virchow coined the term "amyloidosis" derived from the Greek words "amylon" and "eidos" meaning starch-like due to its biochemical properties. ${ }^{10}$ Burrow was the first to describe amyloid deposits in the larynx in 1875.1

Amyloidosis can be categorized as hereditary, primary (when it develops spontaneously), or secondary (secondary to an infectious or chronic inflammatory disease). Primary amyloidosis is further subclassified into a localized form with deposits in a single location or organ such as the larynx, or a generalized form in which deposits are widespread. ${ }^{2}$ Biochemical classification based on the type of protein recognized, has identified 25 types, of which the commonest (in descending order) are the AL (Light chain) type derived from plasma cells containing kappa and lambda immunoglobulin light chains associated with myelomas, amyloidassociated (AA) type which is a non-immunoglobulin protein synthesized in the liver and found in systemic amyloidosis and the amyloid-beta $(A B)$ type with beta- 2 microglobulin deposits in Alzheimer's disease and hemodialysis associated amyloidosis., ${ }^{11,12}$

Localized amyloidosis accounts for $9-15 \%$ of all cases with amyloidosis. ${ }^{8}$ Although the etiopathogenesis of localized amyloidosis remains relatively ambiguous, two hypotheses have been offered. The first resembles a plasma cell dyscrasia with localized production of abnormal light chains by the plasma cells at the site of deposition, while the second which has been considered as the more plausible explanation suggests a failure by the mucosaassociated lymphoid tissue to clear circulating precursor protein deposited due to a breach in the vasculature. ${ }^{13}$ Localized laryngeal amyloidosis is a rare disorder accounting for a mere $0.2-1.2 \%$ of all benign laryngeal masses. ${ }^{1}$

Laryngeal amyloidosis has been observed more frequently in males than in females, with a male:female ratio of 3:2 in studies by Behnoud and Baghbanian and Mitrani and Biller. ${ }^{13,14}$ However, our study identified a female preponderance with a male:female ratio of 1:1.29. Literature shows that individuals aged between 8 years and 80 years have been affected, with the highest incidence observed between 50 years and 70 years of age. ${ }^{1,8,14}$ In our study, however, 
Table 1: Analysis of disease characteristics in relation to the specific anatomical location of the laryngeal lesion

\begin{tabular}{|c|c|c|c|c|c|}
\hline \multirow[b]{2}{*}{ Parameter } & & \multicolumn{3}{|c|}{$\begin{array}{c}\text { Subsite of larynx affected (values expressed as frequency } \\
\text { except where specified) }\end{array}$} & \multirow[b]{2}{*}{ pvalue } \\
\hline & & Supraglottis $(n=8)$ & Glottis $(n=2)$ & Subglottis $(n=6)$ & \\
\hline Mean age (in years) & & $34.63 \pm 2.59$ & $35 \pm 3$ & $36 \pm 10.42$ & 0.94303 \\
\hline \multirow[t]{2}{*}{ Gender } & Male & $6(75 \%)$ & $1(50 \%)$ & $1(16.67 \%)$ & 0.09277 \\
\hline & Female & $2(25 \%)$ & $1(50 \%)$ & $5(83.33 \%)$ & \\
\hline Mean duration of symptoms (in months) & & $7.25 \pm 3.07$ & $7 \pm 1$ & $2.5 \pm 0.5$ & $0.00942^{*}$ \\
\hline \multirow[t]{2}{*}{ Symptoms } & Hoarseness of voice & $8(100 \%)$ & $2(100 \%)$ & 0 & $0.00012^{*}$ \\
\hline & Dyspnea & 0 & 0 & $6(100 \%)$ & \\
\hline \multirow[t]{2}{*}{ Laterality of lesion } & Unilateral & $5(62.5 \%)$ & $2(100 \%)$ & 0 & $0.01189^{*}$ \\
\hline & Bilateral & $3(37.5 \%)$ & 0 & $6(100 \%)$ & \\
\hline \multirow[t]{2}{*}{ Macroscopic lesion type } & Polypoid & $2(25 \%)$ & $2(100 \%)$ & 0 & $0.03187^{*}$ \\
\hline & Infiltrative & $6(75 \%)$ & 0 & $6(100 \%)$ & \\
\hline \multirow[t]{2}{*}{ Immunohistochemical type } & $\mathrm{AL}$ & 7 (87.5\%) & $2(100 \%)$ & $6(100 \%)$ & 1.0 \\
\hline & $\mathrm{AA}$ & $1(12.5 \%)$ & 0 & 0 & \\
\hline \multirow[t]{2}{*}{ Microlaryngoscopic excision } & Cold steel instrumentation & $8(100 \%)$ & $2(100 \%)$ & $1(16.7 \%)$ & \\
\hline & Coblation assisted & 0 & 0 & $5(83.3 \%)$ & \\
\hline Recurrence & 0 & 0 & $1(16.7 \%)$ & & \\
\hline
\end{tabular}

${ }^{*} p<0.05$ is considered as significant
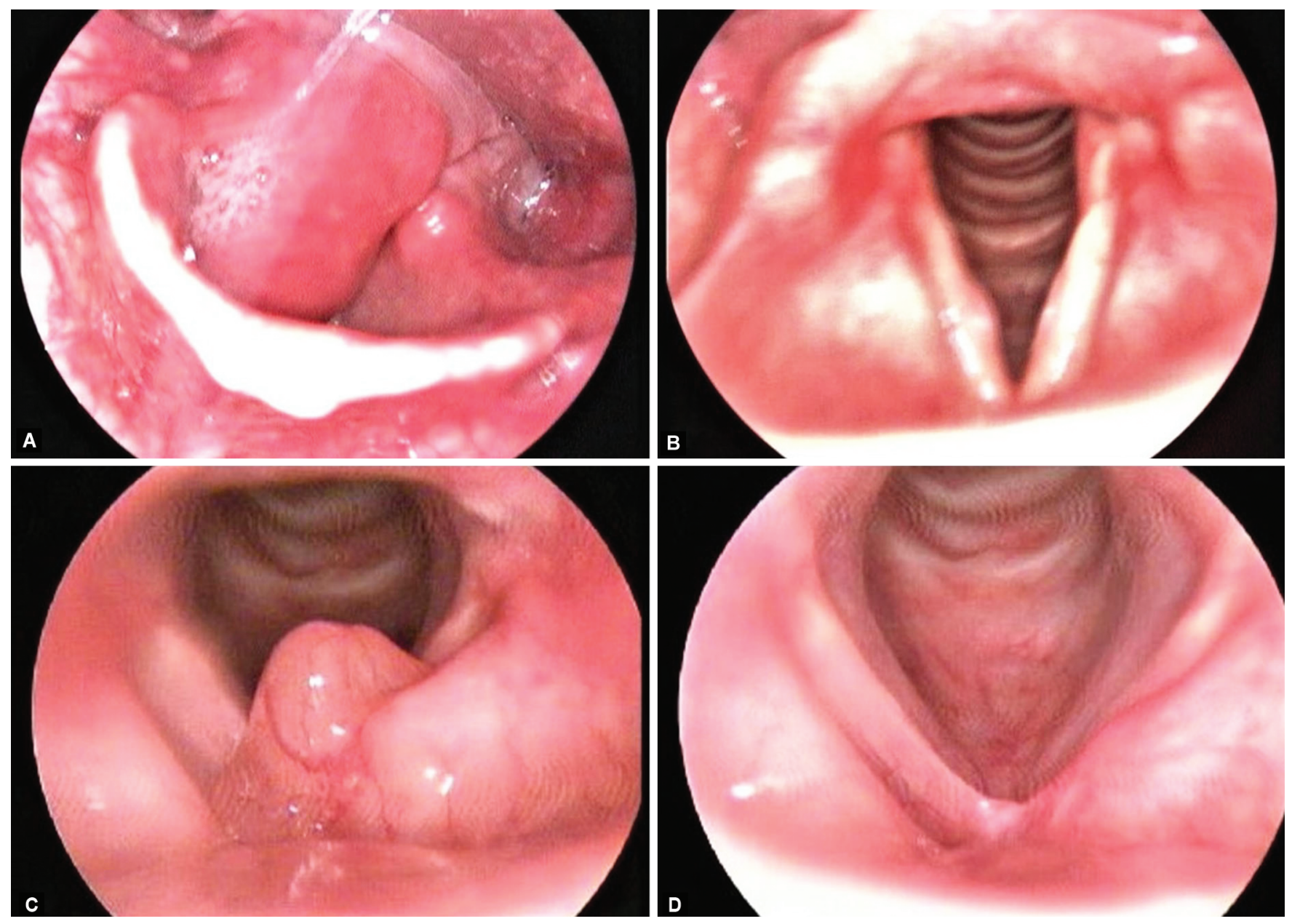

Figs 2A to D: Videolaryngoscopy images show: (A) A polypoid type of lesion in the right half of the supraglottis preoperatively and (B) 1 month following endoscopic excision; (C) A polypoid type of lesion in the left half of the supraglottis preoperatively and (D) 6 months following endoscopic excision 
patients were younger, with $87.5 \%$ of cases aged between 30 years and 40 years. The duration of symptoms in this condition has been recorded as ranging from 6 weeks to 12 months, with an average of 8 months in previous studies. ${ }^{15}$ Similarly, the duration of symptoms in our study was between 2 months and 12 months, with an average of 5.4 months. Symptoms include hoarseness of voice, cough globus, hemoptysis, and varying degrees of dyspnea and stridor, depending on the site and extent of disease, with hoarseness being the most common symptom, as was observed in our study. ${ }^{13}$ Supraglottic and glottic lesions presented with hoarseness of voice and a longer duration of symptoms, whereas subglottic lesions presented with dyspnea and a much shorter duration of symptoms owing to a reduction in airway patency and consequent greater discomfort.

Laryngeal amyloidosis has been known to involve any subsite of the larynx. Conflicting reports in the literature exist as to whether the most common subsite of involvement within the larynx is the true vocal cord as described by Raymond et al. or the supraglottis, namely the ventricle, followed by the false cord as described by Passerotti et al., ${ }^{9,16}$ Our study concurred with the latter by identifying $50 \%$ of cases with supraglottic involvement (of which $100 \%$ were located in the false cord), followed by subglottic involvement in $37.5 \%$ of patients and glottic involvement in $12.5 \%$. Among the cases with glottic involvement, only one case showed true vocal cord involvement, while in the other, the lesion was in the infraglottic region near anterior commissure (within the anatomical limits of the glottis). Though multiple subsites (e.g., glottosupraglottic) together getting involved with amyloid deposits has been mentioned in the literature, ${ }^{11}$ no such case was found in our series.

First described by New in 1919, macroscopically, two types of lesions are observed in cases with laryngeal amyloidosis, i.e., a discrete tumor-like nodular/polypoid lesion and subepithelial infiltrating/granular lesions, as were seen in 25 and $75 \%$ of cases, respectively, in our study. ${ }^{17}$ Comparable with the report on amyloidosis by Balbani et al. ${ }^{18}$ which stated that nodular deposits were seen more often in supraglottic and glottic lesions while subglottic lesions were predominantly infiltrative, our study found that $100 \%$ of subglottic lesions were infiltrative, while $100 \%$ of glottic and $25 \%$ of supraglottic lesions were the polypoid type. With the clinical picture in laryngeal amyloidosis closely resembling conditions such as laryngeal malignancies, granulomatosis with polyangiitis, polyps, and nodules, a high degree of suspicion is required to recognize and diagnose this condition. 7,19,20

Conclusive identification of amyloidosis is dependent on pathological examination of tissue. Routine hematoxylin and eosin staining of the representative tissue shows diffuse, amorphous, acellular eosinophilic deposits in amyloidosis. The gold standard for the confirmation and typing of amyloidosis has been established as tissue biopsy for Congo Red staining showing apple-green birefringence under polarized light and immunohistochemistry respectively, as was performed in our study. ${ }^{5,8}$ Among those with laryngeal amyloidosis, primary isolated amyloidosis of the AL type is significantly more common than systemic amyloidosis involving the larynx. ${ }^{21,22}$ In concurrence with this literature, no evidence of systemic amyloidosis was identified in any of our cases upon evaluation for the same, and $93.75 \%$ of cases were of the AL type.

Identification or exclusion of systemic amyloidosis is crucial in the prognostication of the disease and hence all patients in our study underwent relevant and thorough evaluation in accordance with accepted clinical protocols and consultation with the Department of Internal Medicine at our center, thereby ruling out systemic involvement in $100 \%$ of cases. ${ }^{21}$ With varying clinical courses, the spectrum of treatment for laryngeal amyloidosis ranges from observation to a partial laryngectomy, depending on the type of disease, i.e., the localized disease is known to run a more benign course with slow progression, whereas the systemic forms are notorious for their aggressive nature thereby yielding a poor prognosis. ${ }^{10,13}$ Localized laryngeal amyloidosis is best treated by complete surgical excision. Other therapeutic approaches that have been tried and have yielded variable results are radiotherapy and chemotherapy with agents such as corticosteroids, melphalan, and colchicine. ${ }^{13,23}$

Endolaryngeal excision has been documented as being commonly performed with either cold knife or carbon dioxide laser, with the latter having gained popularity in recent practice owing to minimization of localized scarring and web formation and its consequent effect on the quality of voice or airway luminal diameter. ${ }^{10}$ Little literature exists about the use of coblation in microlaryngoscopic excision of laryngeal amyloidosis, but its success in the treatment of other benign laryngeal lesions such as respiratory papillomatosis has been established. Coblation technology provides combined ablation, resection, coagulation of the soft tissue, and hemostasis. Compared with lasers, coblation is less expensive and does not require specific safety norms such as specialized endotracheal tubes, etc., as it does not run the risk of an airway fire as seen with lasers. ${ }^{24,25}$ In our study, $68.75 \%$ of cases underwent cold steel microlaryngoscopic excision of the lesion, while $31.25 \%$ underwent coblation-assisted microlaryngoscopic excision. All the cases that were subjected to coblation-assisted excision had subglottic lesions (i.e., in five out of six cases with subglottic lesions). Keeping in mind the maintenance of airway luminal patency during the unpredictable healing process following excision which may be accompanied by scarring/webbing, all subglottic lesions being bilateral in our study, underwent staged surgical excision. ${ }^{26}$ All procedures were performed without a tracheostomy. Subjective improvement in symptoms was reported in all cases following surgical intervention. On follow-up in the sixth month, the case with the subglottic lesion who had undergone conventional microlaryngoscopic excision (i.e., one out of six cases), had a recurrence of symptoms, was detected with a similar lesion at the same site, and hence underwent revision microlaryngoscopic excision. Carbon dioxide laser is useful in recurring lesions because it is minimally invasive and preserves laryngeal function. ${ }^{22}$ We used diode laser for recurrent lesion, as carbon dioxide laser and coblator were unavailable in the department (the aforementioned case was one among the oldest in the series). Recurrence was not observed in the remaining $93.75 \%$ of cases on regular follow-up. Due to the high rate of recurrence of these lesions, regular laryngoscopic follow-up is imperative for the early detection of a recurrence, as was the protocol in our cases, and multiple surgical procedures may be necessary for the excision of symptomatic recurrences. ${ }^{21}$

\section{Conclusion}

Laryngeal amyloidosis is a rare clinical entity that can often be overlooked in the differential diagnosis of a laryngeal mass. This necessitates a high index of suspicion clinically based on its short duration of symptoms and easily identifiable appearance on office laryngoscopy. The localized laryngeal disease can be successfully treated by endoscopic excision of the lesion using either the time tested cold steel instrumentation or with laser or with the help of 
coblation, as was successfully performed in our study, leading the way for further research into the expansion of the various tools available in the treatment of this condition. The importance of continued long-term follow-up is emphasized in view of a high predilection for recurrence in amyloidosis.

\section{References}

1. Thompson LD, Derringer GA, Wenig BM. Amyloidosis of the larynx: a clinicopathologic study of 11 cases. Mod Pathol 2000;13(5):528-535. DOI: $10.1038 /$ modpathol.3880092.

2. CelenkF, Durucu C, Baysal E, et.al. Management of upper aerodigestive tract amyloidosis. Ann Otol, Rhinol Laryngol 2013;122(8):535-540. DOI: $10.1177 / 000348941312200810$.

3. Westermark P. Aspects on human amyloid forms and their fibril polypeptides. FEBS J 2005;272(23):5942-5949. DOI: 10.1111/j.17424658.2005.05024.x.

4. Penner CR, Muller S. Head and neck amyloidosis: a clinicopathologic study of 15 cases. Oral Oncol 2006;42(4):421-429. DOI: 10.1016/j. oraloncology.2005.09.010.

5. Picken MM. The pathology of amyloidosis in classification: a review. Acta Haematol 2020;143(4):322-334. DOI: 10.1159/000506696.

6. Gallivan GJ, Gallivan HK. Laryngeal amyloidosis causing hoarseness and airway obstruction. J Voice 2010;24(2):235-239. DOI: 10.1016/j. jvoice.2008.07.006.

7. Muneeb A, Gupta S. Isolated laryngeal amyloidosis mimicking laryngeal cancer. Cureus 2018;10(8):e3106. DOI: 10.7759/cureus. 3106.

8. Ma L, Bandarchi B, Sasaki C, et.al. Primary localized laryngeal amyloidosis: report of 3 cases with long-term follow-up and review of the literature. Archi Pathol Laborat Med 2005;129(2):215-218. DOI: 10.5858/2005-129-215-PLLARO.

9. Passerotti GH, Caniello M, Hachiya A, et al. Multiple-sited amyloidosis in the upper aerodigestive tract: case report and literature review. Rev Bras Otorinolaringol 2008;74(3):462-466. DOI: 10.1590/S003472992008000300024.

10. Alaani A, Warfield AT, Pracy JP. Management of laryngeal amyloidosis. J Laryngol Otol 2004;118(4):279. DOI: 10.1258/002221504323012021.

11. Benning S, Technau-Ihling K, Fisch P, et al. Amyloid tumor of the larynx associated with plasma cell infiltration: differential diagnosis. Ear, Nose Throat J 2004;83(12):839-843. DOI: 10.1177/014556130408301218.
12. Merlini G, Stone MJ. Dangerous small B-cell clones. Blood 2006;108(8):2520-2530. DOI: 10.1182/blood-2006-03-001164.

13. Behnoud F, Baghbanian N. Isolated laryngeal amyloidosis. Iranian J Otorhinolaryngol 2013;25(70):49.

14. Mitrani M, Biller HF. Laryngeal amyloidosis. Laryngoscope 1985;95(11):1346-1347. DOI: 10.1288/00005537-198511000-00010.

15. Nerurkar NK, Sapre AP, Gosavi RS. Primary laryngeal amyloidosis: a discussion of 10 cases with a review of the literature. Int J Phonosurg Laryngol 2018;8(1):52-60. DOI: 10.5005/jp-journals-10023-1158.

16. Raymond AK, Sneige N, Batsakis JG. Amyloidosis in the upper aerodigestive tracts. Ann Otol, Rhinol Laryngol 1992;101(9):794-796. DOI: $10.1177 / 000348949210100915$.

17. New GB. Amyloid tumors of the upper air passages. Laryngoscope 1919;29(6):327-341. DOI: 10.1288/00005537-191906000-00001.

18. Balbani AP, Formigoni GG, Sennes LU, et al. Primary laryngeal amyloidosis in a child. J Otolaryngol Head Neck Surg 1999;28(3):171.

19. Yiotakis I, Georgolios A, Charalabopoulos A, et al. Primary localized laryngeal amyloidosis presenting with hoarseness and dysphagia: a case report. J Med Case Rep 2009;3(1):1-5. DOI: 10.4076/1752-1947-39049.

20. de Queiroz Pimentel C, Lanzarin L, de Oliveira Santana F, et al. Laryngeal primary amyloidosis: differential diagnosis of localized granulomatosis with polyangiitis. Report of two cases. Rheumatica Acta: Open Access 2017;1(1):017-019. DOI: 10.17352/raoa.000004.

21. Pribitkin E, Friedman O, O'Hara B, et al. Amyloidosis of the upper aerodigestive tract. Laryngoscope 2003;113(12):2095-2101. DOI: 10.1097/00005537-200312000-00007.

22. Gillmore JD, Hawkins PN. Amyloidosis and the respiratory tract. Thorax 1999;54(5):444-451. DOI: 10.1136/thx.54.5.444.

23. Skinner M, Anderson JJ, Simms R, et al. Treatment of 100 patients with primary amyloidosis: a randomized trial of melphalan, prednisone and colchicine versus colchicine only. Am J Med 1996;100(3):290-298. DOI: 10.1016/s0002-9343(97)89487-9.

24. Zhou C, Sun B, Wang F, et al. Coblation plus photodynamic therapy (PDT) for the treatment of juvenile onset laryngeal papillomatosis. World J Surg Oncol 2014;12(1):275. DOI: 10.1186/1477-7819-12-275.

25. Zeitels SM, Burns JA. Laser applications in laryngology: past, present and future. Otolaryngol Clin North Am 2006;39(1):159-172. DOI: 10.1016/j.otc.2005.10.001.

26. Hazenberg AJC, Hazenberg BPC, Dikkers FG. Long-term follow-up after surgery in localized laryngeal amyloidosis. Eur Arch Otorhinolaryngol 2016;273(9):2613-2620. DOI: 10.1007/s00405-016-4061-y. 